\title{
New Electron Cyclotron Emission Diagnostic Based Upon the Electron Bernstein Wave
}

\author{
P. C. Efthimion, J. C. Hosea, R. Kaita, R. Majeski, G. Taylor \\ Princeton University Plasma Physics Lab \\ Princeton, NJ 08543
}

Abstract
PECEIVED

MAY 241999

OSTI

Most magnetically confined plasma devices cannot take advantage of standard Electron Cyclotron Emission (ECE) diagnostics to measure temperature. They either operate at high density relative to their magnetic field or they do not have sufficient density and temperature to reach the blackbody condition. The standard ECE technique measures the electromagnetic waves emanating from the plasma. Here we propose to measure electron Bernstein waves (EBW) to ascertain the local electron temperature in these plasmas. The optical thickness of EBW is extremely high because it is an electrostatic wave with a large $\mathrm{k}_{\mathrm{i}}$. One can reach the blackbody condition with a plasma density $\approx 10^{11} \mathrm{~cm}^{-3}$ and $T_{e} \approx 1 \mathrm{eV}$. This makes it attractive to most plasma devices. One serious issue with using EBW is the wave accessibility. EBW may be accessible by either direct coupling or mode conversion through an extremely narrow layer $(\approx 1-2 \mathrm{~mm})$ in low field devices.

The advent of electron cyclotron emission (ECE) diagnostics for magnetic fusion plasmas has made it possible for the time evolution of the electron temperature profile to be measured with moderately priced microwave and millimeter wave technology [1-3]. ECE measurements with excellent temporal and spatial resolution have facilitated the study of plasma transport and MHD fluctuations. Two criteria must be satisfied for the application of ECE diagnostics: satisfactory wave damping and accessibility. The accessibility criteria limits the application to moderate $\beta$ plasmas where $\Omega_{\mathrm{ce}}>\omega_{\mathrm{pe}}$ for the ordinary mode (O-mode) fundamental and $\omega_{\mathrm{pe}}{ }^{2} / \omega^{2}<1-\Omega_{\mathrm{ce}} / \omega$ for the second harmonic extraordinary mode (X-mode), where $\Omega_{\mathrm{ce}}$ is the electron cyclotron frequency, $\omega_{\mathrm{pe}}$ is the electron plasma frequency, and $\omega$ is the wave frequency. The wave damping requires the optical thickness $\tau>2$ for the ECE to be at the blackbody level and proportional to the electron temperature. Consequently, only high temperature plasmas $\left(T_{e}>1 \mathrm{keV}\right)$ with low $\beta$ such as found in tokamaks and stellerators can meet the criteria. Many magnetized plasmas cannot utilize the technique. The inverse process of heating a plasma near the electron cyclotron frequency is governed by the same issues. 


\section{DISCLAIMER}

This report was prepared as an account of work sponsored by an agency of the United States Government. Neither the United States Government nor any agency thereof, nor any of their employees, make any warranty, express or implied, or assumes any legal liability or responsibility for the accuracy, completeness, or usefulness of any information, apparatus, product, or process disclosed, or represents that its use would not infringe privately owned rights. Reference herein to any specific commercial product, process, or service by trade name, trademark, manufacturer, or otherwise does not necessarily constitute or imply its endorsement, recommendation, or favoring by the United States Government or any agency thereof. The views and opinions of authors expressed herein do not necessarily state or reflect those of the United States Government or any agency thereof. 


\section{DISCLAIMER}

Portions of this document may be illegible in electronic image products. Images are produced from the best available original document. 


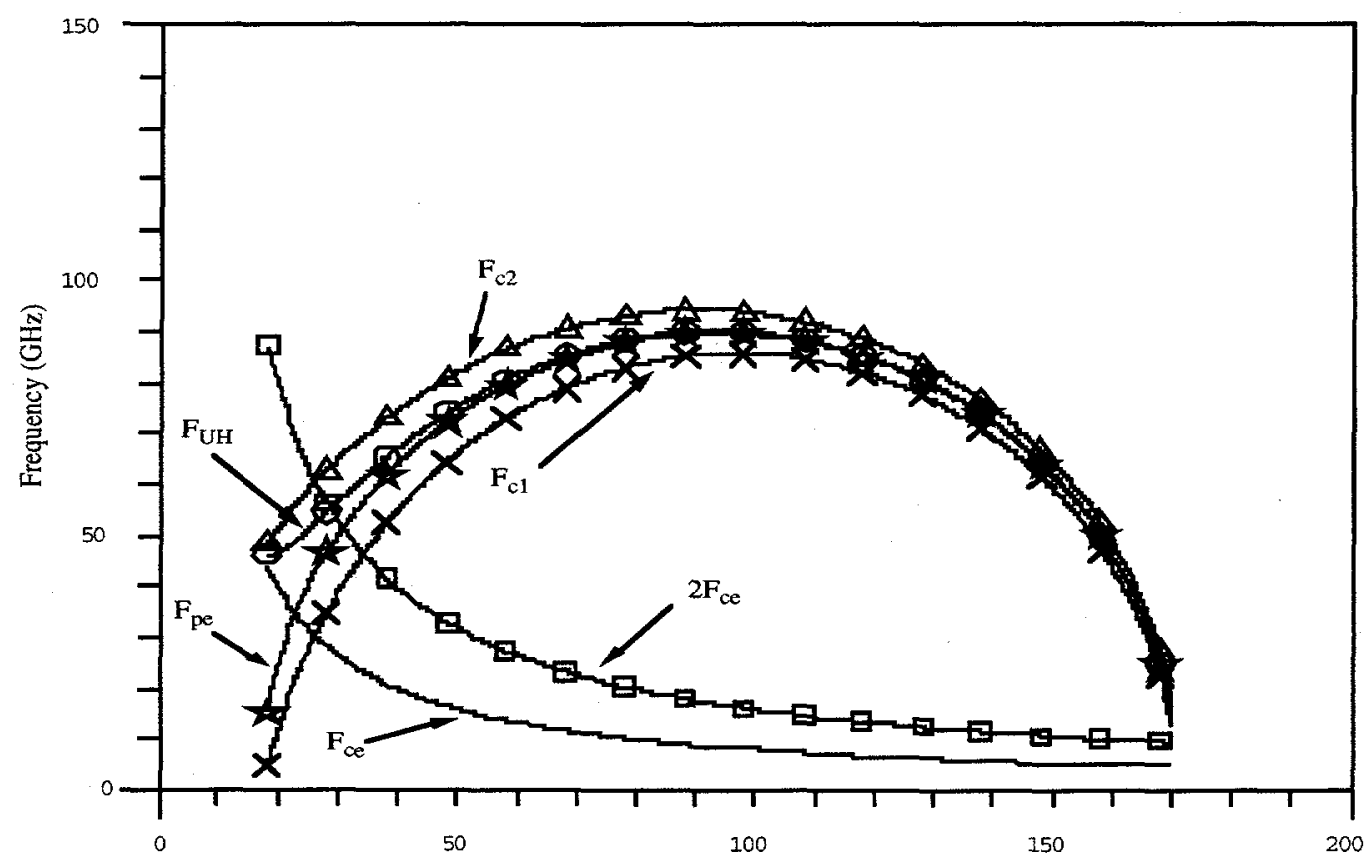

Major Radius (cm)

Fig. 1 Characteristic frequencies in NSTX versus major radius. Fce is the electron cyclotron frequency, FUH is the upper hybrid frequency, and $\mathrm{Fcl}$ and $\mathrm{Fc} 2$ are the upper X-mode cutoff frequencies, respectively.

A new toroidal configuration, the spherical torus, is being investigated as an alternate magnetic confinement concept. The first such device, START [4], has achieved high densities with a modest toroidal magnetic field. Core densities of $3 \times 10^{13} \mathrm{~cm}^{-3}$ are routine and $10^{14} \mathrm{~cm}^{-3}$ has been achieved with magnetic fields $B_{\phi}=0.2-0.6 \mathrm{~T}$. Similar conditions are expected for NSTX [5]. Such conditions translate into $\omega_{\mathrm{pe}} / \Omega_{\mathrm{ce}} \gg 1$. The characteristic frequencies for NSTX are shown in Fig. 1. Note the plasma frequency is larger than the first few electron cyclotron frequencies at every radius except near the inner plasma edge. The cutoff for the extraordinary mode is even larger than the plasma frequency. Therefore, at each radius of the NSTX plasma except near the inner plasma edge, the plasma cannot support electromagnetic waves for the first five electron cyclotron harmonics and electromagnetic radiation is not generated from these harmonics. However, electron Berstein waves are generated and propagate in the plasma as will be described later. Although very high harmonic electromagnetic radiation is accessible it is far from the blackbody condition necessary for the measurement of temperature or for effective electron heating at microwave frequencies.

From the electrostatic dispersion relation for a collisionless hot plasma in Bekefi [7] and Stix [8] one can easily calculate the real and imaginary wave number for this range of frequencies:

$\operatorname{Re}\left[\mathrm{k}_{\mathrm{perp}}\right] \approx\left(4 / \pi^{1 / 2}\right)^{1 / 3}\left(\Omega_{\mathrm{ce}} / \mathrm{V}_{\mathrm{T}}\right)\left(\omega_{\mathrm{pe}}{ }^{2} /\left(\omega^{2}-\left(\Omega_{\mathrm{ce}}-\Delta \Omega_{\mathrm{ce}}\right)^{2}\right)^{1 / 3}\right.$

$\operatorname{Im}\left[k_{\mathrm{perp}}\right] \approx A \pi^{1 / 2} \omega_{\mathrm{pe}}{ }^{2} \Omega_{\mathrm{ce}}^{2} / \operatorname{Re}\left[\mathrm{k}_{\mathrm{perp}}{ }^{2}\right] \mathrm{V}_{\mathrm{T}}^{3}$ 
where $\Delta \Omega_{\mathrm{ce}}=\Omega_{\mathrm{ce}} \mathrm{V}_{\mathrm{T}}^{2} / \mathrm{c}^{2}, \mathrm{~V}_{\mathrm{T}}$ is the electron thermal velocity, and $\mathrm{A}=\mathrm{F}\left(\omega, \Omega_{\mathrm{ce}}, \mathrm{T}_{\mathrm{e}}\right)$ and is defined in reference 6 .

From these equations, the wave propagation can be calculated from $\operatorname{Re}\left[\mathrm{k}_{\text {perp }}\right]$. For $\omega=\omega_{\mathrm{ce}}$, the optical thickness, $\tau$, can be calculated from $\operatorname{Im}\left[\mathrm{k}_{\text {perp }}\right]$ for various machines. For PLT, the optical thickness is on the order of 10,000. Interestingly enough the optical thickness for NSTX is 3000 . For NSTX, the entire plasma diameter is highly absorptive to EBW, and the emission easily meets the blackbody condition for measuring electron temperature, $\tau>2$. The plasmas of these machines should also be optically thick for second and third harmonic electron Berstein waves. The high absorptivity makes EBW extremely attractive for electron cyclotron resonant heating and current drive on these machines. Furthermore, the high absorptivity for EBW permits any magnetized plasma with an electron density, $n_{\mathfrak{e}} \geq 10^{11} \mathrm{~cm}^{-3}$ and an electron temperature, $T_{\mathfrak{e}} \geq 1 \mathrm{eV}$ to satisfy the blackbody criterion. Therefore, many magnetized plasmas meet the blackbody condition which allows EBW electron temperature diagnostic measurements, EBW heating and current drive to be employed.

Simple accessibility arguments indicate that there are both direct and mode conversion scenarios for accessing EBW emission from the electron cyclotron layer. The characteristic frequencies for NSTX near the plasma edge in Fig. 3 indicate the possibility of direct coupling with EBW. The coupling distance through the X-mode cutoff layers $\left(\mathrm{F}_{\mathrm{c} 1}, \mathrm{Fc} 2\right)$ and upper hybrid layer is on the order of a few millimeters allowing for direct coupling (evanescent) as is achieved with lower hybrid current drive with a slow wave antenna. One major difference is the $\mathrm{EBW}$ is an $\mathrm{X}$-mode ( $\mathrm{E}$ perpendicular to $\mathrm{B}$ ) while the lower hybrid wave is an $\mathrm{O}$-mode (E parallel to $\mathrm{B}$ ). Direct coupling will require an antenna that matches the low phase velocity of the electrostatic EBW similar to the coupling scheme for lower hybrid waves. A multiple element antenna array is normally used to create a low phase velocity $k$-spectrum for lower hybrid wave coupling by adjusting the phase between the antenna elements [9]. However, electron temperature measurements may not require a narrow bandwidth $\mathrm{k}$-spectrum and thus, a two element antenna may be sufficient for coupling to EBW. In the microwave range of frequencies waveguides are the antenna elements. Direct coupling would be favorable because the coupling efficiency would not be very sensitive over the range of the electron cyclotron frequencies of interest. To achieve efficient direct coupling the electric field from the antenna has to extend across the cutoffs and resonances shown in Fig. 3. This will require the antenna placed close to the last closed flux surface where the plasma density $n_{e} \geq 5 \times 10^{11} \mathrm{~cm}^{-3}$. Alternately, one mode conversion scenario has the EBW propagate to the upper hybrid layer and mode convert to an electromagnetic X-mode which propagates out of the plasma. This scenario has been studied by Bers [10]. Another mode conversion scheme has the EBW propagating to the upper hybrid layer, mode converting into an X-mode, tunnel through the cutoff layer and mode converting to the O-mode at a turning point. This last coupling scheme was successfully used to heat W7-X above the density limit for the launched O-mode [11].

The optical thickness calculations indicate that EBW physics, and electron 


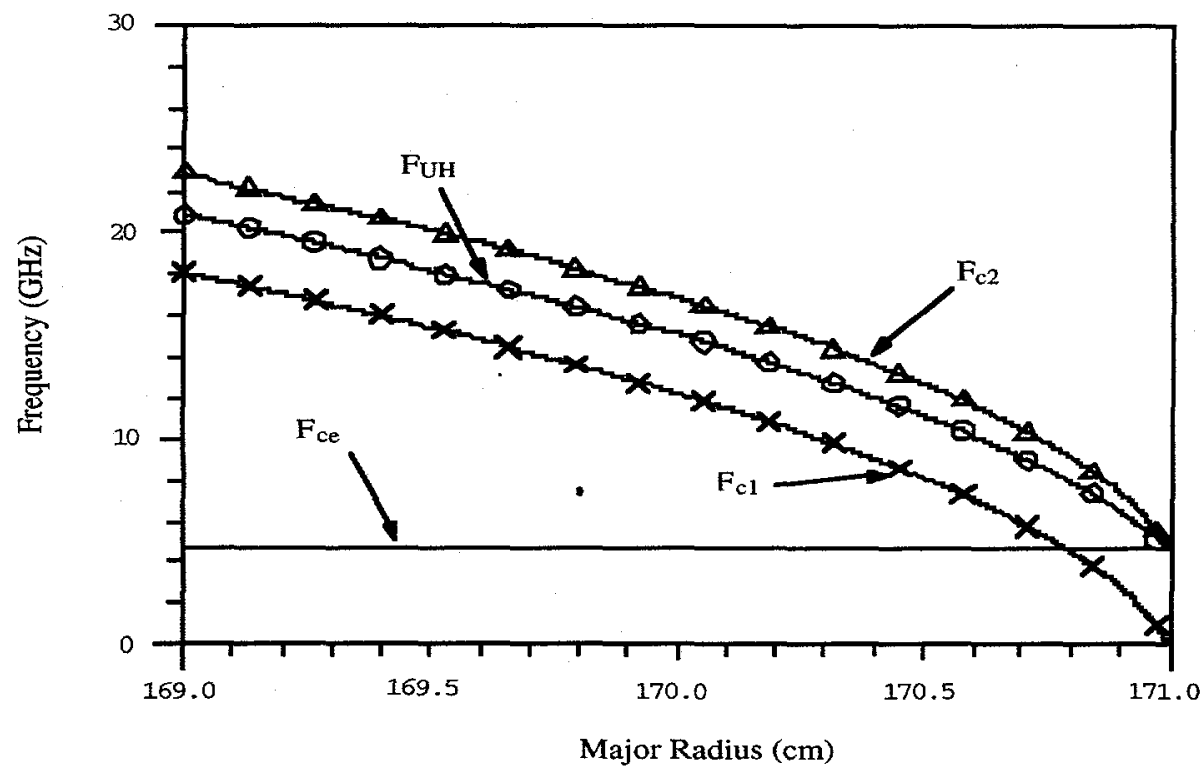

Fig. 2 Characteristic frequencies for NSTX near the edge major radius. Characteristic frequencies are within a few millimeters of the plasma edge indicating the possibility of direct coupling of the EBW to an antenna at the plasma edge.

temperature proof-of principle measurements can be studied on CDX-U. The CDX-U plasma has an optical thickness $\approx 300$ and can be accessed with simple electromagnetic and electrostatic antennas. The emission frequencies for CDX-U are below $20 \mathrm{GHz}$. A heterodyne EBW radiometer is being installed on CDX-U. A successful program on CDX-U will support the installation of a similar instrument on NSTX to measure the electron temperature. This instrumentation would be used to study MHD and provide profile measurements for confinement studies. Furthermore, the emission measurements on CDX-U and then on NSTX are necessary steps for the serious consideration of EBW heating and current drive. The high absorptivity of EBW allows for the consideration of electron temperature measurement, electron heating, and current drive on modest magnetized plasmas $\left(n_{e} \geq 10^{11} \mathrm{~cm}^{-3}\right.$ and $\left.T_{e} \geq 1 \mathrm{eV}\right)$.

*Work supported by DOE contract No. DE-AC02-76-CH-03073.

[1] Efthimion, P. C., et. al., Rev. Sci. Inst. 70, 1018 (1999).

[2] Bornatici, M, et. al., Nuclear Fusion 23, 1153 (1983).

[3] Costley, A. E., et. al., Phys. Rev. Lett. 33, 758 (1974).

[4] Gates, D. A., et. al., Phys. of Plasmas 5, 1775 (1998).

[5] Spitzer, J., et. al., Fusion Technology 30, 1337 (1996).

[6] J. Hosea, V. Arunasalam, and R. Cano, Phys. Rev. Lett. 39, 408 (1977).

[7] G. Bekefi, Radiation Processes in a Plasma, Wiley, New York (1966).

[8]T. H. Stix, The Theory of Plasma Waves, McGraw-Hill, New York (1962).

[9] S. Bernabei, et. al., Phys. Rev. Lett., 34866 (1975).

[10] K. C. Wu, A. K. Ram, and A. Bers, Proc. Am. Phys. Soc. 41, 1425 (1996).

[11] H. P. Laqua, et. al., Phys. Rev. Lett. 78, 3467 (1997). 